\title{
Autoantibodies of $\beta$-adrenergic and M2 cholinergic receptors: atrial fibrillation in hyperthyroidism
}

\author{
Guanghong Jia $\cdot$ James R. Sowers
}

Received: 6 February 2015/Accepted: 13 February 2015/Published online: 21 February 2015

(C) Springer Science+Business Media New York 2015

Thyroid hormone (TH) effects on the heart are multiple and are largely mediated by triiodothyronine acting through nuclear-based thyroid receptors [1]. Elevated TH levels can increase gene transcription of cardiomyocyte proteins and exert electrophysiological effects either directly through nuclear thyroid receptors or indirectly by stimulating the sympathoadrenergic system [1-5]. Clinical manifestations of hyperthyroidism include atrial fibrillation (AF) with its attendant risks of heart failure and stroke in patients with hyperthyroidism [5]. Graves' disease (GD), an autoimmune disorder is the most common cause of hyperthyroidism, is associated with an increased risk of $\mathrm{AF}$ and attendant increases in heart failure and stroke [1-5] (Fig. 1). The prevalence of $\mathrm{AF}$ in patients with hyperthyroidism is $10-15 \%$ and this prevalence increases with age [5-8].

To set the stage for understanding how hyperthyroidism promotes $\mathrm{AF}$, we will briefly review the effects of $\mathrm{TH}$ on the heart. TH plays a key role in the modulation of heart rate, heart rhythm, blood pressure, cardiac contractility, and cardiac hypertrophy by controlling the cardiac gene expression, such as alpha myosin heavy chain fusion

\section{G. Jia $\cdot$ J. R. Sowers $(\bowtie)$}

Division of Endocrinology, Diabetes, and Metabolism, Department of Medicine, Diabetes Cardiovascular Center, University of Missouri School of Medicine, D109 Diabetes Center HSC, One Hospital Drive, Columbia, MO 65212, USA e-mail: sowersj@health.missouri.edu

G. Jia $\cdot$ J. R. Sowers

Harry S. Truman Memorial Veterans Hospital, Columbia, MO, USA

\section{J. R. Sowers}

Department of Medical Pharmacology and Physiology, University of Missouri School of Medicine, Columbia, MO, USA
(MHC- $\alpha$ ), MHC- $\beta$, sarcoplasmic reticulum calcium-activated ATPase (SERCA), $\beta 1$ adrenergic receptor, phospholamban (PLB), calcium $\left(\mathrm{Ca}^{2+}\right)$ transporter proteins, cardiac troponin I, atrial natriuretic peptide (ANP), adenylyl cyclase (IV and $\mathrm{V}$ ), and the sodium $(\mathrm{Na})^{+}-\mathrm{Ca}^{2+}$ antiporter [1]. TH also has effects on SERCA, which is important for both normal systolic and diastolic function $[1,8]$. For example, TH promotes increases in SERCa ${ }^{2+}$ ATPase and the ryanodine channel, and decreases phosphorylation of PLB and inhibits SERCa ${ }^{2+}$ pump activity. These SERCa ${ }^{2+}$ and PLB changes can be linked to a decrease in the rate of diastolic relaxation [8]. TH regulates the expression of specific cardiac genes related to heart rhythm, such as plasma membrane sodium potassium $\left(\mathrm{K}^{+}\right)$ ATPase and voltage-activated K1 channel genes including $\mathrm{Kv} 4.2, \mathrm{Kv} 4.3$, and $\mathrm{Kv} 1.5$ [1, 8]. The signaling pathways such as mitogen-activated protein kinase (MAPK) and protein kinase B (Akt) have been reported to be involved in the cardiac functional changes induced by hyperthyroidism. For example, activated serum kinases contribute to increased sinoatrial activity, lowering of the threshold for atrial activity, and shortened atrial repolarization resulting in heart rate increases in thyrotoxic-dilated cardiomyopathy [1, 8]. Hyperthyroidism also affects expression of cardiac connexins, which can "talk" to each other, directly exchange ions and messenger molecules between adjacent cardiomyocytes and play important roles in the promotion of arrhythmogenesis [1]. Thus, TH exerts a broad range of effects on cardiac development, growth, metabolism, and electrophysiological changes.

Factors such as increased automaticity and enhanced triggered activity in response to elevated $\mathrm{TH}$ levels promote arrhythmogenic activity [1] (Fig. 1). One recent study reported that inflammation is also involved in the hyperthyroidism-induced AF [7]. To this point, hyperthyroidism 
Fig. 1 Thyroid hormone, autoantibodies activation, and inflammation act synergistically to develop atrial fibrillation in Grave disease

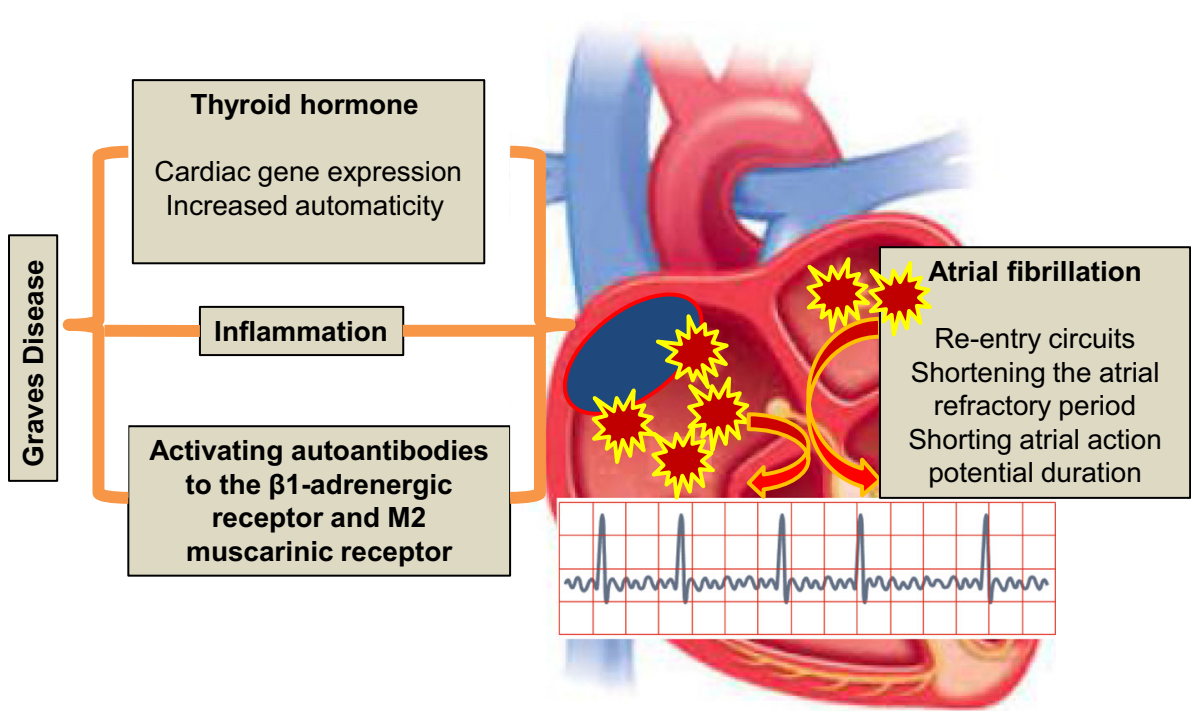

and associated elevated serum levels of interleukin-8, tumor necrosis factor alpha, and C-reactive protein increase the interstitial inflammation in the atrioventricular node and the atrial-ventricular conducting tissue [8]. These data suggest that inflammation is an important trigger factor in hyperthyroidism-induced AF. Indeed, the pulmonary vein is regarded as an important source of re-entry related ectopic beats, initiation of paroxysmal $\mathrm{AF}$ and focal $\mathrm{AF}$ its persistence [7, 8]. In this regard, excess $\mathrm{TH}$ alters the electrophysiological activity of pulmonary vein cardiomyocytes and leads to shortening of the action potential duration in the atrial myocardium and thereby facilitates formation of multiple re-entry circuits. Studies conducted under voltage clamp conditions showed that $\mathrm{TH}$ accelerates diastolic depolarization and pacemaker activity by an upregulation of the $\mathrm{Na}^{+}-\mathrm{Ca}^{2+}$ exchanger $[1,7,8]$. Several ionic current channels may contribute to pacemaker activity in this tissue, including the delayed rectifier potassium current, both the L-type and T-type calcium currents and a background $\mathrm{Na}^{+}$current, which results in shortening of action potential duration $[1,8]$. The electrogenic $\mathrm{Na}^{+}-$ $\mathrm{Ca}^{2+}$ exchanger, triggered as a result of sarcoplasmic reticulum $\mathrm{Ca}^{2+}$ release, may also contribute to the initial phases of diastolic depolarization in the sinoatrial node. Thus, elevated TH levels favor re-entry and the persistence of AF by shortening the atrial refractory period.

An interesting and important study in this issue of Endocrine [9] demonstrates a novel pathophysiological role for receptor-activating autoantibodies in the pathogenesis of AF in Graves (hyperthyroid) patients [9] (Fig. 1). It was observed that the mean $\beta 1$-adrenergic receptor ( $\beta 1 \mathrm{AR})$ and M2 muscarinic receptor (M2R) autoantibody activity was elevated in both GD groups but higher in those with AF than those with sinus rhythm, suggesting activating autoantibodies to $\beta 1 \mathrm{AR}$ and M2R are prevalent in Graves' patients and significantly contribute to the pathogenesis of AF. This relationship was established by examining the relationship of activating autoantibodies to atrial tachyarrhythmias in two separate groups of patients with autoimmune hyperthyroidism versus those with subacute thyroiditis. Indeed, M2R activation in serum from autoimmune hyperthyroid patients was shown to decrease the action potential duration and effective refractory period, while $\beta 1 \mathrm{AR}$ activation lead to intracellular SERCA calcium loading abnormalities. These actions promoted AF through facilitating rapid electrical firing in cardiac atrial tissues by local autonomic nerve stimulation resulting in AF $[9,10]$. Thus, it is likely that activating autoantibodies and TH act synergistically in some patients with GD to induce rapid depolarization in pulmonary veins to induce AF. The importance of the present study is to provide evidence that patients with Graves develop significant autoantibody titers of $\beta 1 \mathrm{AR}$ and $\mathrm{M} 2 \mathrm{R}$, and that the synergistic arrhythmogenic effect of receptor-activating autoantibodies and TH facilitate development of AF. Accordingly, these unique activating autoantibodies may play a role in the initiation and maintenance of AF. It is likely that future therapies targeting reduction of these pathological autoantibodies may improve or prevent the persistence of tachyarrhythmias in this patient population.

In summary, these data suggest a new concept in the pathogenesis of AF in the hyperthyroid patient; namely, the highly specific receptor activity inherent with $\beta 1 \mathrm{AR}$ and $M 2 R$ autoantibodies may increase site-specific vulnerability of atrial cells as triggers and/or to demonstrate an enhanced substrate susceptibility to generate and/or sustain various atrial tachyarrhythmias. Thus, autoantibodies of $\beta 1 \mathrm{AR}$ and $\mathrm{M} 2 \mathrm{R}$ are independent predictors of $\mathrm{AF}$ in 
patients with hyperthyroidism. Further studies are necessary to develop safe and clinically effective autoantibodies modulators that target AF in this patient population.

Acknowledgments The authors gratefully acknowledge Brenda Hunter for editorial assistance. The research of the authors is supported by funding from the National Institutes of Health (R01HL73101 and R01-HL107910 to J.R.S.) and the Department of Veterans Affairs Biomedical Laboratory Research and Development Merit (0018 to J.R.S.).

Conflict of interest The authors have no conflicts of interest to disclose.

\section{References}

1. I.M. Grais, J.R. Sowers, Thyroid and the heart. Am. J. Med. 127, 691-698 (2014)

2. G.J. Kahaly, W.H. Dillmann, Thyroid hormone action in the heart. Endocr. Rev. 26, 704-728 (2005)

3. I. Klein, S. Danzi, Thyroid disease and the heart. Circulation 116, 1725-1735 (2007)

4. B. Biondi, G.J. Kahaly, Cardiovascular involvement in patients with different causes of hyperthyroidism. Nat. Rev. Endocrinol. 6, 431-443 (2010)
5. S. Ertek, A.F. Cicero, Hyperthyroidism and cardiovascular complications: a narrative review on the basis of pathophysiology. Arch. Med. Sci. 9, 944-952 (2013)

6. S. Marrakchi, F. Kanoun, S. Idriss, I. Kammoun, Arrhythmia and thyroid dysfunction. Herz (2014). doi:10.1007/s00059-014-4123-0

7. M. Ozaydin, A. Kutlucan, Y. Turker, B. Koroglu, A. Arslan, B.A. Uysal, D. Erdogan, E. Varol, A. Dogan, Association of inflammation with atrial fibrillation in hyperthyroidism. J. Geriatr. Cardiol. 9, 344-348 (2012)

8. Z.Q. Sun, K. Ojamaa, T.Y. Nakamura, M. Artman, I. Klein, W.A. Coetzee, Thyroid hormone increases pacemaker activity in rat neonatal atrial myocytes. J. Mol. Cell. Cardiol. 33, 811-824 (2001)

9. A. Galloway, H. Li, M. Vanderlinde-Wood, M. Khan, A. Benbrook, C. Liles, C. Zillner, V. Rao, M.W. Cunningham, X. Yu, D.C. Kem, Activating autoantibodies to the $\beta 1,2$-adrenergic and M2 muscarinic receptors associate with atrial tachyarrhythmias in patients with hyperthyroidism. Endocrine (2014). doi:10.1007/ s12020-014-0495-4

10. S. Stavrakis, X. Yu, E. Patterson, S. Huang, S.R. Hamlett, L. Chalmers, R. Pappy, M.W. Cunningham, S.A. Morshed, T.F. Davies, R. Lazzara, D.C. Kem, Activating autoantibodies to the beta- 1 adrenergic and $\mathrm{m} 2$ muscarinic receptors facilitate atrial fibrillation in patients with Graves' hyperthyroidism. J. Am. Coll. Cardiol. 54, 1309-1316 (2009) 